\title{
Efficacy and Safety of Miniscalpel Acupuncture on Knee Osteoarthritis
}

\section{- A randomized controlled pilot trial -}

\author{
Seungah Jun', Jung Hee Lee ${ }^{1}$, Han Mi Gong', Seong Hun $\mathrm{Choi}^{2}$, Min Hwang $\mathrm{Bo}^{3}$, Mi \\ Suk Kang ${ }^{4}$, Geon-Mok Lee ${ }^{5}$, Hyun-Jong Lee ${ }^{1}$, Jae Soo Kim ${ }^{1 *}$
}

\begin{abstract}
'Department of Acupuncture \& Moxibustion medicine, College of Korean medicine, Daegu Haany University, 136, Sincheondong-ro, Suseong-gu, Daegu, 706-828, Korea

2 Department of Anatomy and Histology, College of Korean medicine, Daegu Haany University, 136, Sincheondong-ro, Suseong-gu, Daegu, ${ }^{3}$ Department of Oriental Ophthalmology and Otolaryngology and Dermatology, College of Korean medicine, Daegu Haany University, 136 , Sincheondong-ro, Suseong-gu, Daegu, 706-828, Korea

${ }^{4}$ Department of Acupuncture \& Moxibustion, College of Korean medicine, Gachon University, 1342 Seongnam-daero, Seongnam 13120, Korea

${ }^{5}$ Department of Acupotomy, LeeGeonmok Wonli Korean Medicine Hospital, Seoul 137-829, Korea
\end{abstract}

\section{Key Words}

acupuncture, knee osteoarthritis, miniscalpel acupucnture

\section{Abstract}

Objectives: We investigated the efficacy and safety of miniscalpel acupuncture (MA) for knee osteoarthritis (KOA) in an assessor-blinded randomized controlled pilot trial; this would provide information for a largescale randomized controlled trial.

Methods: Participants $(n=24)$ were recruited and randomly allocated to the MA group (experimental) or acupuncture group (control). The MA group received treatment once a week for 3 weeks (total of 3 treatments), while the acupuncture group received treatment two times per week for 3 weeks (total of 6 treatments). The primary outcome was pain as assessed by a visual analogue scale (VAS). The secondary outcomes (intensity of current pain, stiffness, and physical function) were assessed using the short-form McGill Pain Questionnaire (SF-MPQ) and Western Ontario and McMaster Universities Osteoarthritis Index (WOMAC). Assessments were

Received: Feb 09, 2108 Reviewed: Jul 26, 2018 Accepted: Aug 07,2018

$@$ This is an Open-Access article distributed under the terms of the Creative Common Attribution Non-Commercial License (http://creativecommons.org/licenses/by-nc/4.0/) which permits unrestricted noncommercial use, distribution, and reproduction in any medium, provided the original work is properly cited.

( This paper meets the requirements of KS X ISO 9706, ISO 9706-1994 and ANSI/NISO Z39.48-1992 (Permanence of Paper). performed at baseline, 1,2 , and 3 during treatment and at week 5 ( 2 weeks after the end of treatment).

Results: Of the 24 participants, 23 completed the study. Both groups showed significant improvements in VAS, SF-MPQ, and WOMAC. However, there were no significant differences between the MA and acupuncture groups. No serious adverse event occurred and blood test results were within normal limits.

Conclusion: Our results suggest that although both MA and acupuncture provide similar effects with regard to pain control in patients with KOA, MA may be more effective in providing pain relief because the same relief was obtained with fewer treatments. A large-scale clinical study is warranted to further clarify these findings.

\section{Introduction}

Knee osteoarthritis (KOA) is the most common form of arthritis [1]. Due to the complexity of the knee joint, it is prone to damage. Medications such as non-steroidal anti-inflammatory drugs and glucosamine are commonly used to relieve symptoms [2]. In severe cases,

*Corresponding Autho

Jae Soo Kim, K. M.D. Department of Acupuncture \& Moxibustion medicine, Daegu Oriental hospital of Daegu Haany University, 136, Sincheondong-ro, Suseong-gu, Daegu, 706-828, Korea.

Tel: +82-53-770-2112 Fax: +82-53-770-2055

E-mail: jaice@daum.net 
topical analgesics, intra-articular injections of sodium hyaluronate, and surgical treatment such as osteotomy and knee joint replacement may be necessary $[3,4]$. Current conventional therapy aims to reduce pain and physical disability, and prevent structural failure. Pharmacological therapy may provide pain relief but serious adverse effects can occur especially to elderly patients [5]. Although surgical treatment may be more beneficial than other existing treatments, risks including infection and other side effects can occur. Because of the limited medical treatments available and the risks associated with surgery, patients with $\mathrm{KOA}$ are seeking alternative therapies [6, 7].

Miniscalpel acupuncture (MA) is a new subtype of acupuncture that is effective in treating chronic soft tissue injuries such as adhesions and contractures [8]. Miniscalpel acupuncture is more therapeutic than traditional acupuncture in the treatment of chronic musculoskeletal pain [9]. The aim of MA is to recover the kinetic state of soft tissue by disrupting adhesions and separating the attached tissues [10]. When compared to traditional acupuncture, MA has the advantage of rapid recovery and pain reduction [11]. However, there is little scientific evidence supporting the use of MA for KOA. In China, a number of studies on the use of MA have been published; however, rigorous scientific studies are rare. Therefore, we designed a protocol for an assessor-blinded, randomized controlled pilot trial to compare the effects of MA with acupuncture in patients with KOA. The study was designed to obtain information for a future large-scale trial and provide information about the feasibility of using MA in patients with KOA.

\section{Materials and Methods}

\subsection{Study design}

This protocol followed the Declaration of Helsinki and Korean Good Clinical Practice (KGCP). The study was carried out at Daegu Oriental Hospital of Daegu Haany University, Daegu, Republic of Korea. The trial was registered with the Korean Clinical Research Information Service (CRIS) registry (KCT0001899). Written informed consent was obtained from each participant before any treatment was given. The sample size was fixed at 24 participants. The study design is presented in Figure 1. The study lasted 5 weeks. Participants were randomly allocated into two groups: control (acupuncture) and experimental (MA). Participants in the control group received acupuncture and electro-acupuncture two times weekly for 3 weeks. Participants in the experimental group received MA one time weekly for 3 weeks. Assessments were performed at baseline and at 1, 2, 3, and 5 weeks.

\subsection{Participants}

Participants with KOA were recruited from December 2015 to August 2016 through advertisements on the hospital website and on bulletin boards. Eligibility determined by one researcher and was based on the results of the phys- ical examination and radiographs. The inclusion criteria were: (1) age between 40-80 years; (2) diagnosed with KOA according to American College of Rheumatology criteria; (3) stop taking KOA medications at least one week before starting the study; and (4) radiographic classification on the Kellgren-Lawrence scale of 2-4. The exclusion criteria were: (1) previous knee surgery; (2) intra-articular injection or prolotherapy within the past three months; (3) senile dementia, impaired cognitive function, severe psychiatric, or psychologic disorders; (4) steroid or nonsteroidal anti-inflammatory medications to treat other diseases; (5) post-traumatic osteoarthritis; (6) allergic skin disease, skin ulcer, or skin infection; (7) pregnant, lactating, or planning to become pregnant; (8) insulin dependent diabetes mellitus; (9) cerebral, cardiovascular, or renal disease; (10) use of thrombolytic, antiplatelet, or anticoagulant medications; (11) abnormal renal or hepatic function on laboratory tests; (12) pacemaker; and (13) deemed ineligible by the recruiting physician.

\subsection{Randomization}

Random numbers were generated by an independent statistics professional through computerized block randomization using SPSS version 19.0 for Windows (release 14.0K; SPSS Inc. Armonk, NY, USA). Sealed opaque assignment envelopes were used for allocation concealment. Participants were randomly assigned to the control or experimental group. The researcher evaluating the outcome measure was blinded to the treatment allocation.

\subsection{Intervention}

If participants had both knee pain, only one knee with more pain was treated. No other interventions such as moxibustion, exercises or lifestyle advice were done.

\section{MA}

The MA points were as follows [12-14]: quadriceps tendon (EX-LE2); patella ligament (EX-LE210); medial collateral ligament (LR8); lateral collateral ligament (GB33); medial and lateral region of the patella; and medial and lateral sides of the quadriceps tendon. Sterile disposable $0.5 \mathrm{~mm} \times 50 \mathrm{~mm}$ MA needles (DongBang Acupuncture Inc., Korea) were used. After sterilizing the MA insertion sites with ethanol (80\%, Firson Inc., Korea) and povidone iodine (10\%, Firson Inc., Korea), a physician trained in western medicine provided local anesthesia with lidocaine $(1 \%, 3 \mathrm{ml})$ using a $27 \mathrm{~g} \times 1.5$ inch disposable needle. For injection of local anesthetic, the knee was flexed 30 to 45 degree. A Korean medical doctor (KMD), licensed by Ministry of Health and Welfare, performed the MA. At the end of treatment, the KMD who performed the MA evaluated the participants for adverse events or bleeding.

\section{Acupuncture}

The acupuncture points were as follows [15-17]: unilateral ST34, SP9, SP10, EX-LE4, EX-LE2, LR8, ST36, GB33, and 
EX-LE5. Sterile disposable $0.3 \mathrm{~mm}$ x $40 \mathrm{~mm}$ acupuncture needles (DongBang Acupuncture Inc., Korea) were used. A needle was inserted into each acupuncture point. After all the needles were inserted, an electro-acupuncture device (Suzuki Iryoki Co., Ltd., Tokyo, Japan) was used to stimulate two acupuncture points (unilateral LR8, EX-LE2). The needles remained in place for $20 \pm 5$ minutes.

\subsection{Outcome measurement}

The primary outcome was severity of pain measured on a visual analogue scale (VAS). The VAS is a $10-\mathrm{cm}$ measurement instrument used to determine the severity of pain. Each subject rated knee pain on a scale of 0 to 10 , where 0 indicates the absence of pain and 10 indicates the worst pain imaginable $[18,19]$.

The secondary outcomes of intensity of current pain, stiffness, and physical function were assessed by the shortform McGill pain questionnaire (SF-MPQ) and Western Ontario and McMaster Universities Osteoarthritis Index (WOMAC). The SF-MPQ has sensory, affective, and evaluative dimensions $[20,21]$. The scale is based on 15 descriptors, including 11 items in the sensory domain and 4 items in the emotional domain. Each item is scored on an intensity scale from 0 to 3 , with $0=$ none; $1=$ mild; 2 $=$ moderate; and $3=$ severe. Pain scores are obtained by summing the individual scores of all 15 items, with higher scores indicating more severe pain. The Present Pain Index (PPI) measures the severity of current pain. Scores range from 0 to 5 , with $0=$ no pain; $1=$ mild; $2=$ discomforting; 3 = distressing; 4 = horrible; and 5 = excruciating pain. The WOMAC is a widely used standardized questionnaires to evaluate osteoarthritis of the knee and hip, including pain, stiffness, and physical functioning of the joints [5]. The WOMAC consists of 24 items divided into 3 subscales. The index measures five items for pain (score range 0 to 20 ), two for stiffness (score range 0 to 8 ), and 17 for physical function (score range 0 to 68) [22]. Higher scores on the WOMAC indicate worse pain, stiffness, and functional limitation. Both questionnaires were administered at baseline, 1, 2, 3, and 5 weeks.

\subsection{Safety}

Prior to the study and at week 5, blood was obtained for a complete blood count, erythrocyte sedimentation rate, aspartate aminotransferase, alanine aminotransferase, blood urea nitrogen, creatinine, prothrombin time, partial thromboplastin time, C-reactive protein, and electrolytes. The occurrence of swelling, redness, itching, peripheral neuritis, hemorrhage, bruising, and pain were recorded as the adverse events. Subjects were asked to voluntarily report adverse effects and the researchers confirmed the occurrence by an interview or physical examination.

\subsection{Data analysis}

PData were analyzed based on the Statistics Guidelines for Clinical Trials [23] and using SPSS 19.0 for Windows (Version 14.0; SPSS Inc., Armonk, NY, USA). A p-value of
$<0.05$ was considered statistically significant.

The last observation carried forward method was used for missing data. Demographic variables and clinical characteristics were compared between groups using a two-sample t-test or Mann-Whitney U, if the date were normally distributed and not continuous. The chi-square test was used for categorical data.

A repeated-measures analysis of variance (ANOVA) was used to assess between-group differences with regard to changes in the scores of the VAS, SF-MPQ, and WOMAC.

\section{Results}

\subsection{Baseline characteristics}

The baseline characteristics and outcome measurements are shown in Table 1. There were no significant differences between the groups.

\subsection{Enrollment rate and dropout ratio}

Figure 1 shows the study design flow chart. A total of 30 individuals were screened to enroll 24 participants. Of the individuals screened, 2 did not meet the radiographic inclusion criteria; one did not return after screening, and three withdrew the consent before randomization. Twenty-three subjects completed the trial. One subject in the control group withdrew consent after the first visit for personal reasons The compliance rate for the remaining 23 subjects was $100 \%$.

\subsection{Primary outcome}

There was a significant decrease in the VAS scores for both groups when baseline scores were compared to scores from weeks 1, 2, 3, and 5. Both groups had a significant improvement in VAS scores; however, there were no significant differences between groups (Table 2). There was no interaction between time and group (Table 2).

\subsection{Secondary outcomes}

The SF-MPQ and WOMAC scores at weeks 1, 2, 3, and 5 were significantly reduced compared to baseline $(\mathrm{p}<0.05)$. However, there were no significant differences between groups (Table 3 ).

\subsection{Adverse events}

Two subjects in the MA group and four subjects in the acupuncture group experienced bruises. One subject reported increased intensity of knee pain; however, the relationship with MA was unclear. All laboratory test results were within the normal range. 
Table 1 Baseline characteristics and outcome measurements for participants

\begin{tabular}{|c|c|c|c|c|}
\hline \multirow{2}{*}{\multicolumn{2}{|c|}{ Variable }} & \multicolumn{2}{|c|}{ Group \pm SD } & \multirow{3}{*}{ p-value } \\
\hline & & $\begin{array}{l}\text { Experiment } \\
\text { group }\end{array}$ & Control group & \\
\hline \multirow{2}{*}{ Sex, $n(\%)$} & Male & $1(8.3)$ & $4(33.3)$ & \\
\hline & Female & $11(91.7)$ & $8(66.7)$ & 0.132 \\
\hline \multicolumn{2}{|c|}{ Mean age, years } & $63.67 \pm 9.745$ & $66.17 \pm 5.04$ & 0.441 \\
\hline \multicolumn{2}{|c|}{ Weight, mean \pm SD } & $61.31 \pm 8.674$ & $63.98 \pm 10.97$ & 0.516 \\
\hline \multicolumn{2}{|c|}{ Height, mean \pm SD } & $152.93 \pm 6.56$ & $158.17 \pm 10.51$ & 0.158 \\
\hline \multicolumn{2}{|c|}{ VAS } & $5.53 \pm 2.15$ & $4.90 \pm 1.72$ & 0.443 \\
\hline \multirow[b]{2}{*}{ SF-MPQ } & PPI & $2.00 \pm 0.85$ & $2.09 \pm 0.54$ & 0.761 \\
\hline & $\begin{array}{c}\text { Description } \\
\text { scale }\end{array}$ & $11.67 \pm 6.24$ & $10.09 \pm 4.83$ & 0.504 \\
\hline \multicolumn{2}{|c|}{ WOMAC } & $39.83 \pm 18.90$ & $30.73 \pm 18.71$ & 0.259 \\
\hline
\end{tabular}

Values are expressed as means \pm standard deviations

SD: Standard deviation

VAS: Visual analogue scale

SF-MPQ: Short-form McGill pain questionnaire

PPI: Present pain intensity

WOMAC: Western Ontario and McMaster Universities Osteoarthritis Index 
Table 2 Changes in visual analogue scale score for Knee osteoarthritis

\begin{tabular}{|c|c|c|c|c|c|c|c|}
\hline \multirow{2}{*}{ Group } & \multicolumn{3}{|c|}{ Time, mean \pm SD } & \multicolumn{2}{c|}{ p-value } \\
\cline { 2 - 7 } & Screening & Week 1 & Week 2 & Week 3 & Week 5 & Time & Time $\times$ Group \\
\hline MA group & $7.14 \pm 1.63$ & $5.53 \pm 2.15^{*}$ & $4.74 \pm 2.04^{* *}$ & $3.72 \pm 2.70^{* *}$ & $3.33 \pm 2.67^{* *}$ & \multirow{2}{*}{$0.000^{\dagger}$} & $0.946^{\dagger}$ \\
\hline Acupuncture group & $6.60 \pm 1.68$ & $4.90 \pm 1.72^{*}$ & $4.19 \pm 1.64^{* *}$ & $3.46 \pm 2.08^{*}$ & $2.67 \pm 2.17^{* *}$ & & \\
\hline
\end{tabular}

Values represent mean \pm SD.

$\mathrm{P}$ values between baseline measurement and measurements at $1,2,3$ and 5 weeks in each group: ${ }^{*} \mathrm{p}<0.05 ;{ }^{* *} \mathrm{p}<.0 .01$.

$\dagger:$ Repeated-measures ANOVA

SD: Standard deviation

MA: Miniscalpel acupuncture

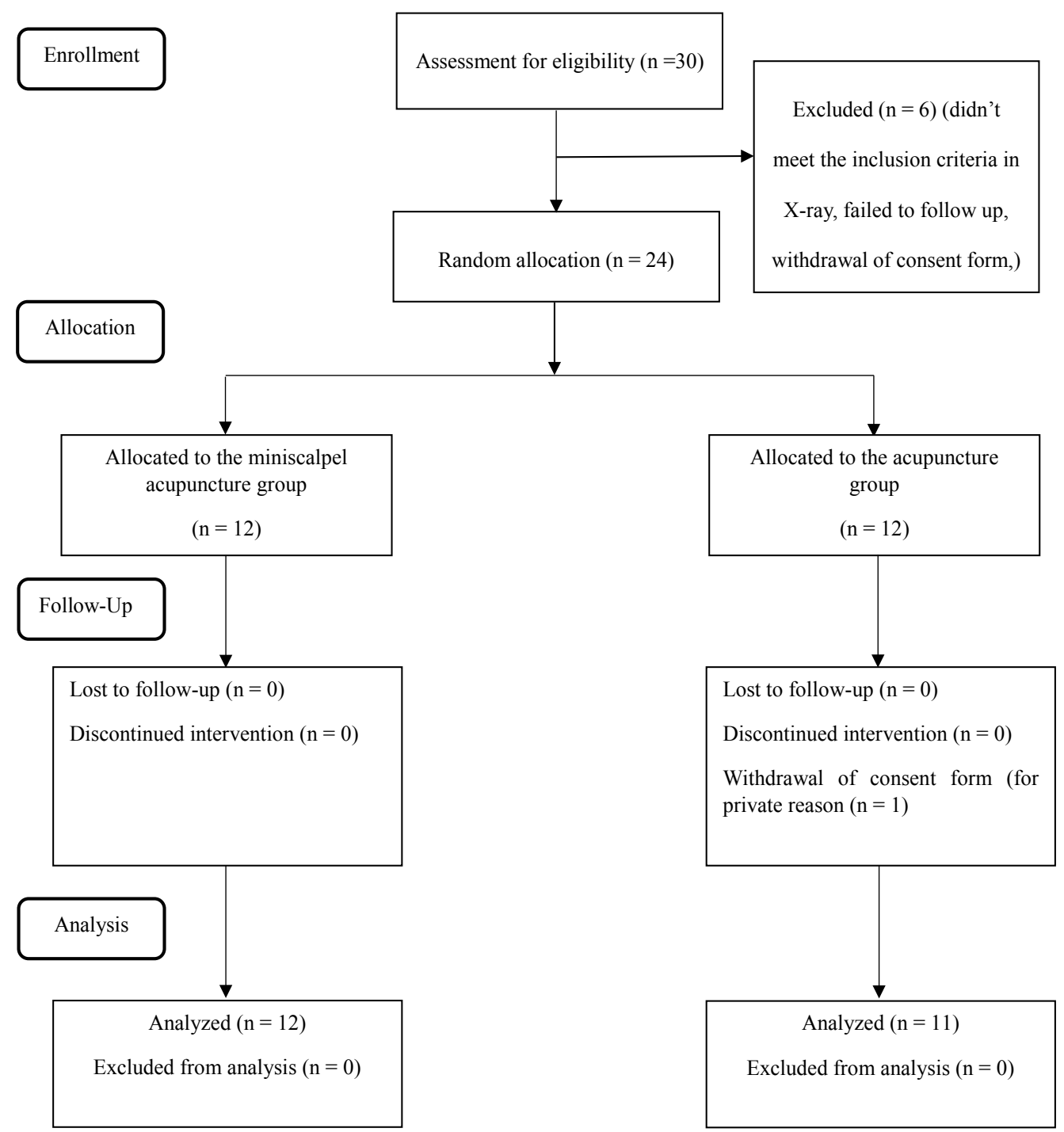

Figure 1 Flow chart for our randomized controlled pilot trial on the efficacy and safety of miniscalpel acupuncture for degenerative osteoarthritis 
Table 3 Changes in short form McGill pain questionnaire and Western Ontario and McMaster Universities Osteoarthritis Index scores for knee osteoarthritis

\begin{tabular}{|c|c|c|c|c|c|c|c|}
\hline & AC group & $3.00 \pm 0.78$ & $2.09 \pm 0.54 * *$ & $1.55 \pm 0.52 *$ & $1.55 \pm 0.52^{* *}$ & $1.45 \pm 0.52 * *$ & $0.000^{\dagger}$ \\
\hline \multirow{2}{*}{$\begin{array}{c}\text { SF-MPQ- } \\
\text { Descriptive scale }\end{array}$} & MA group & $21.92 \pm 7.75$ & $11.67 \pm 6.24 * *$ & $12.00 \pm 6.97 * *$ & $10.67 \pm 8.47 * *$ & $10.50 \pm 5.96^{* *}$ & $0.001^{\dagger}$ \\
\hline & AC group & $19.18 \pm 8.98$ & $10.09 \pm 4.83^{* *}$ & $9.45 \pm 6.20^{* *}$ & $8.45 \pm 6.44^{* *}$ & $8.91 \pm 6.70^{* *}$ & $0.001^{\dagger}$ \\
\hline \multirow[t]{2}{*}{ WOMAC } & MA group & $55.58 \pm 20.24$ & $39.83 \pm 18.90^{* *}$ & $38.17 \pm 19.85^{*}$ & $29.83 \pm 16.71 * *$ & $30.25 \pm 15.20^{*}$ & $0.002^{\dagger}$ \\
\hline & AC group & $44.00 \pm 14.40$ & $30.73 \pm 18.71 *$ & $27.91 \pm 18.96^{*}$ & $26.09 \pm 20.74 * *$ & $22.55 \pm 17.31^{*}$ & $0.000^{\dagger}$ \\
\hline
\end{tabular}

Values represent mean \pm SD.

$\dagger:$ Repeated-measures ANOVA

P values between baseline measurement and measurements at $1,2,3$ and 5 weeks in each group: ${ }^{*} \mathrm{p}<0.05 ;{ }^{*} \mathrm{p}<.0 .01$.

MA: miniscalpel acupuncture; AC: acupuncture

SF-MPQ: short form McGill pain questionnaire

WOMAC: Western Ontario and McMaster Universities Osteoarthritis Index.

\section{Discussion}

Miniscalpel acupuncture is a new acupuncture technique using a sharp knife at the tip of the needle and combining the effects of microsurgery and acupuncture [24]. In Korea the use of MA is increasing. There have been no randomized controlled studies that compare the effects of MA and acupuncture for KOA in Korea. Although MA is widely used in Korea, few randomized controlled trials have been performed. Case reports have described the use of MA in the treatment of musculoskeletal conditions, including partial tear of supraspinatus tendon [25], lumbar herniated intervertebral disc [26], carpal tunnel syndrome [27], and cervicogenic headaches [28]. We designed this study based on the hypothesis that MA is superior to acupuncture for the treatment of chronic degenerative knee pain.

Of the individuals recruited, $80 \%$ agreed to participate in the study. Although there were no serious adverse events, the sample size was small and safety should be carefully considered for future large-scale trials.

After treatment, there was a significant decrease in pain in both groups although there were no differences between groups. These findings are inconsistent with other studies suggesting that MA is more effective than acupuncture for the treatment KOA pain $[29,30]$. In a case report, a patient with degenerative osteoarthritis who did not responded to acupuncture had positive result after MA treatment [30]. However, the difference in the number and frequency of treatment is worth discussing. The MA and acupuncture group appeared to have similar treatment effects but the MA was performed once a week while acupuncture was performed twice a week. Although the acupuncture group had more treatments than MA group, there was no difference between groups, suggesting that the treatment effects of MA are more powerful than acupuncture. The MA group had similar treatment effects with fewer treatments. Miniscalpel acupuncture treatment for patients with chronic pain may be more economic and less time consuming. Miniscalpel acupuncture is more invasive than traditional acupuncture. The small knife at the tip of the needle makes the treatment more painful, and local anesthesia is required. Because MA is invasive and provides a strong stimulus, some participants complained of stiff and dull pain for few days after treatment. Prescribing analgesics for two or three days after treatment might improve patient satisfaction.

Few studies have investigated the mechanism of action of MA, which is considered to work via both acupuncture and the knife. Osteoarthritis of the knee results when matrix degradation is greater than matrix synthesis. Chondrocytes control extracellular matrix (ECM) synthesis [31]. Pro-inflammatory cytokines such as interleukin (IL)-1 $\beta$, IL-6, and tumor necrosis factor (TNF)- $\alpha$ cause inflammation and increased ECM degradation [32]. By inhibiting type II collagen gene expression in articular chondrocytes, IL- $1 \beta$ is the primary cytokine mediating cartilage destruction. Both TNF- $\alpha$ and IL- $1 \beta$ have a role in downregulating synthesis of type II collagen [33-35]. One study of KOA from China showed a greater decrease in IL-1 $\beta$, IL-6, and TNF- $\alpha$ 
in the MA treatment compared to the acupuncture group [29]. This study suggests a possibility that MA may be an effective treatment for KOA. The mechanism of action of MA in KOA has not been elucidated, and future studies should evaluate the mechanism of action in more detail.

In previous randomized controlled trial of KOA [13], the result was examined according to Kellgren-Lawrence grade. MA showed effectiveness in Kellgren-Lawrence grade 3 and 4 . Grade 1 and 2 had no significant effectiveness compared with acupuncture treatment. However previous study did not used detailed or various scales in evaluation. In this pilot study, MA treatment has been performed regardless of Kellgren-Lawrence grade. Also only total result has been calculated to see the effectiveness of MA treatment. In further studies, examining result according to Kellgren-Lawrence grade would be recommended. Also in this pilot study, the same treatment has been done regardless of severity of KOA condition. Also, dividing treatment depending on grade can be considered.

\section{Conclusion}

This pilot study provides information for the design of full-scale trial comparing effect of MA with acupuncture for the treatment of pain in patients with KOA. This pilot study has provided the feasibility and sample size for a fullscale trial. Although there were no significant differences between groups, the effects of MA may be better than acupuncture because the MA group had fewer treatments than the acupuncture group.

\section{Acknowledgement}

This study is supported by a grant from the Ministry of Health \& Welfare, Korea. (CIMI-15-01-09)

\section{Conflict of interest}

The authors declare that there are no conflicts of interest.

\section{Ethics approval}

This protocol has been approved by IRB of Daegu Oriental Hospital of Daegu Haany University, Daegu, Republic of Korea. (IRB approval number DHUMC-D-16001)

\section{References}

1. Berman BM, Singh BB, Lao L, Langenberg P, Li H, Hadhazy $\mathrm{V}$, et al. A randomized trial of acupuncture as an adjunctive therapy in osteoarthritis of the knee. Rheumatology (Oxford). 1999;38:346-54.

2. Bateman DN, Kennedy JG. Non-steroidal anti-inflammatory drugs and elderly patients. BMJ. 1995;310:817-

8.
3. Manek NJ. Medical management of osteoarthritis. Mayo Clin Proc. 2001;76:533-9.

4. Zhang W, Moskowitz RW, Nuki G, Abramson S, Altman $\mathrm{RD}$, Arden $\mathrm{N}$, et al. OARSI recommendations for the management of hip and knee osteoarthritis, Part I: Critical appraisal of existing treatment guidelines and systematic review of current research evidence. Osteoarthritis and Cartilage. 2007;15:981-1000.

5. McConnell S, Kolopack P, Davis AM. The Western Ontario and McMaster Universities Osteoarthritis Index (WOMAC): a review of its utility and measurement properties. Arthritis care \& research. 2001;45:453-61.

6. McGettigan P, Henry D. Cardiovascular risk and inhibition of cyclooxygenase: a systematic review of the observational studies of selective and nonselective inhibitors of cyclooxygenase 2. JAMA. 2006;296:1633-44.

7. Blower A, Brooks A, Fenn G, Hill A, Pearce M, Morant S, et al. Emergency admissions for upper gastrointestinal disease and their relation to NSAID use. Aliment Pharmacol Ther. 1997;11:283-91.

8. Guo C, Liu N, Li X, Sun H, Hu B, Lu J, et al. Effect of acupotomy on nitric oxide synthase and beta-endorphin in third lumbar vertebrae transverse process syndrome model rats. Journal of Traditional Chinese Medicine. 2014;34:194-8.

9. Ma C, Wu S, Li G, Xiao X, Mai M, Yan T. Comparison of miniscalpel-needle release, acupuncture needling, and stretching exercise to trigger point in myofascial pain syndrome. Clin J Pain. 2010;26:251-7.

10. Lee G, Kim D, Kim H, Yeom S, Kim H, Kim D. The study on the effect of acupotomy in lumbar HIVD. The Journal of Korean Acupuncture \& Moxibustion Medicine society. 2008;25:183-90.

11. Kim S. Clinical characteristics of poor responders to acupotomy and safety pretreatment management. The Acupuncture. 2008;25:117-25.

12. Lee GM, Kim DH, Kim HW, Cho NG, Choi YS, Kim HS, et al. The Cilnical Study on the Effect of Acupotomy Osteoarthritis on knee Using Five-Finger Placed to Marking Position. The Acupuncture. 2008;25:197-202.

13. Lin M, Li X, Liang W, Liu J, Guo J, Zheng J, et al. Needle-knife therapy improves the clinical symptoms of knee osteoarthritis by inhibiting the expression of inflammatory cytokines. Experimental and therapeutic medicine. 2014;7:835-42.

14. The Acupuncture Compilation Committee. The Acupuncture and Moxibustion(Sang). Seoul. In: Anonymous : JipMoonDang. 2008;441-483.

15. Shin SY, Seo DK, Kim SY, Seo JC, Seo YJ, Lee YJ, et al. The Effect of Chinemys reevesii Gray Pharmacopuncture for Women with Knee Osteoarthritis. The Acupuncture. 2015;32:163-73.

16. Min W, Yeo S, Kim E, Song HS, Koo S, Lee J, et al. Comparison of warm-needling and acupuncture for knee osteoarthritis: a randomized controlled trial. Korean Journal of Acupuncture. 2013;30:64-72.

17. Jung HC, Jeong SH. Clinical Study of Knee Joint Osteoarthritis Patients on the Effect of Korean Traditional Medicine Treatment with Acupuncture, Cupping, Physical Therapy and Ganghwalijetong-yeum(Qianghuochutong-yin) : Case Series. The Journal of the So- 
ciety of Korean Medicine Diagnostics. 2015;19:125-32.

18. Revill S, Robinson J, Rosen M, Hogg M. The reliability of a linear analogue for evaluating pain. Anaesthesia. 1976;31:1191-8.

19. Carlsson AM. Assessment of chronic pain. I. Aspects of the reliability and validity of the visual analogue scale. Pain. 1983;16:87-101.

20. Melzack R. The short-form McGill pain questionnaire. Pain. 1987;30:191-7.

21. Burckhardt CS. The use of the McGill Pain Questionnaire in assessing arthritis pain. Pain. 1984;19:305-14.

22. Roos, M Klässbo, LS Lohmander, EM. WOMAC Osteoarthritis Index: Reliability, validity, and responsiveness in patients with arthroscopically assessed osteoarthritis. Scand J Rheumatol. 1999;28:210-5.

23. Food and Drug Administration. Guidance for industry: E9 statistical principles for clinical trials. Food and Drug Administration: Rockville, Maryland, USA; 1998.

24. Ma C, Wu S, Li G, Xiao X, Mai M, Yan T. Comparison of miniscalpel-needle release, acupuncture needling, and stretching exercise to trigger point in myofascial pain syndrome. Clin J Pain. 2010;26:251-7.

25. Kim HS, Heo WY, Hyun MK, Kim JS, Gang IA. Four case of partial tear of supraspinatus tendon treated by acupotomy combined oriental medical treatments. The Acupuncture. 2014;31:167-76.

26. Kim HS, Kim SY, Kim HJ, Kim ES, Kim YI. The Effect of Acupotomy on Lumbar Herniated Intervertebral Disc: Report of a Case Series. The Acupuncture. 2015;32:18595.

27. Kim JI, Kim HS, Park GN, Jeon JH, Kim JH, Kim YI. Miniscalpel Needle Therapy with Integrative Korean Medical Treatment for Carpal Tunnel or Tarsal Tunnel Syndrome: Case Series of Three Patients. The Acupuncture. 2017;34:139-52.

28. Jun S, Lee JH, Gong HM, Chung YJ, Kim JR, Park CA, et al. The Effects of Miniscalpel Acupuncture on Cervicogenic Headache: A report of three cases. The Acupuncture. 2017;34:131-8.

29. Lin M, Li X, Liang W, Liu J, Guo J, Zheng J, et al. Needle-knife therapy improves the clinical symptoms of knee osteoarthritis by inhibiting the expression of inflammatory cytokines. Experimental and therapeutic medicine. 2014;7:835-42.

30. Park MS, Oh SJ, Lee JH, Jun SA, Gong HM, Choi SH, et al. Miniscalpel Acupuncture Treatment on a Knee Degenerative Osteoarthritis Patient, Who does not Responded to Acupuncture Treatment. The Acupuncture. 2016;33:161-8.

31. Dieppe PA, Lohmander LS. Pathogenesis and management of pain in osteoarthritis. The Lancet. 2005;365:965-73.

32. Yuan P, Liu D, Chu X, Hao Y, Chao Z, Qiang Q. Effects of Preventive Administration of Juanbi Capsules on TNF- $a$ IL-1 And IL-6 Contents of Joint Fluid in the Rabbit with Knee Osteoarthritis. Journal of Traditional Chinese Medicine. 2010;30:254-8.

33. Fernandes JC, Martel-Pelletier J, Pelletier J. The role of cytokines in osteoarthritis pathophysiology. Biorheology. 2002;39:237-46.

34. Goldring $\mathrm{MB}$, Berenbaum F. The regulation of chon- drocyte function by proinflammatory mediators. Clin Orthop. 2004;427:S37-46.

35. Smith RL, Allison AC, Schurman DJ. Induction of Articular Cartilage Degradation by Recombinant Interleukin la and 1 $\beta$. Connect Tissue Res. 1989;18:307-16. 